\title{
Changes in cognitive function and related brain regions in chronic benzene poisoning: a case report
}

\author{
Jiaojiao $\mathrm{Hu}^{1} \wedge$, Enyan $\mathrm{Yu}^{1}$, Zhengluan Liao ${ }^{2}$ \\ ${ }^{1}$ Department of Psychiatry, The Cancer Hospital of the University of Chinese Academy of Sciences (Zhejiang Cancer Hospital), Institute of Basic \\ Medicine and Cancer (IBMC), Chinese Academy of Sciences, Hangzhou, China; ${ }^{2}$ Department of Psychiatry, Zhejiang Provincial People's Hospital of \\ Hangzhou Medical College, Hangzhou, China \\ Correspondence to: Enyan Yu. No. 1, East Banshan Road, Gongshu District, Hangzhou, China. Email: yuenyan@aliyun.com.
}

\begin{abstract}
To discuss the changes in cognitive function and related brain regions in patients with chronic benzene poisoning. Few studies have explored the damage to cognitive function that occurs in benzene toxic encephalopathy. It is important to identify early in the course of disease whether cognitive dysfunction is caused by benzene poisoning so that disease prognosis and appropriate treatment can be determined. We reported on the chronic benzene poisoning of a 41-year-old Han Chinese woman. The patient had graduated from primary school, and she had a cheerful and diligent personality. She had performed painting work for more than five years, and her primary work involved painting swivel chairs. The primary reasons she attended the psychiatric clinic were loss of appetite, she had experienced fatigue for more than 2 months, and she had had memory loss for a month. These symptoms seriously impacted the patient's daily life and ability to work. The patient's husband expressed concern that she could not recognize acquaintances, could not find her way home, and had lost approximately $5 \mathrm{~kg}$ per month over two months. We analyzed changes in this chronic benzene poisoning patient's cognitive function with cognitive function assessments and magnetic resonance imaging (MRI). Measurements were taken on presentation to hospital, during the patient's hospitalization, and three months following discharge. Long-term exposure to benzene can damage the central nervous system. However, it is difficult to recognize when cognitive impairment is caused by chronic benzene poisoning, as it rarely presents with a decline in cognitive function as the primary clinical manifestation. Atypical symptoms, such as decreased immune function and gastrointestinal issues, may be the first symptoms to appear, and these atypical symptoms are difficult to detect in the early stages of disease. Regular screening of high-risk groups is required to significantly reduce the incidence of systemic damage caused by benzene poisoning.
\end{abstract}

Keywords: Benzene poisoning; cognitive dysfunction; magnetic resonance imaging (MRI)

Submitted Jul 31, 2020. Accepted for publication Nov 11, 2020.

doi: $10.21037 / \mathrm{atm}-20-6597$

View this article at: http://dx.doi.org/10.21037/atm-20-6597

\section{Introduction}

Benzene is a chemical commonly used in industry and agriculture and is an established human carcinogen (1). This chemical is also widely used in the manufacture of products such as nitrobenzene spices, paint, printing ink, rubber, resin, et cetera. Benzene is extremely poisonous to humans and exerts a strong lipotropic effect (2). Benzene vapor can be inhaled, entering the body through the respiratory tract, and it can also be absorbed through the skin at normal temperatures. Long-term exposure to benzene vapor can damage the hematopoietic system $(3,4)$, immune system (5), and nervous system (6), which leads

\footnotetext{
^ ORCID: 0000-0001-8705-1238.
} 
to leukopenia, decreased platelets, infection, hemorrhage, cognitive dysfunction, et cetera $(7,8)$. Chronic benzene poisoning has a long disease course and is difficult to treat; there is no effective therapy for the systemic damage it causes. However, if patients can be identified and correctly diagnosed in a timely manner, symptoms can be treated early and further disease progression avoided.

It has been reported that occupational exposure to organic solvents is a risk factor for cognitive impairment (9-11). Chlorinated solvents, petroleum solvents, benzenes, and nonbenzene aromatics are used in paints, binders, skimming, and pesticides, and these chemicals are commonly associated with occupational exposure to solvents. The estimated prevalence of exposure to these solvents in the labor force of industrialized countries is $8 \%$ (12). Evident in chronic toxic encephalopathy, chemical solvents affect the central nervous system, impacting cognitive functions such as attention, processing speed, and motor performance $(13,14)$. If exposure levels remain low, memory and attention problems may improve or cease to progress when the exposure is stopped $(15,16)$. Attention deficits and intellectual impairment, however, have been observed in populations exposed to high levels of solvents. Acute cases may result in permanent neurological impairment.

The effect of organic solvents on cognitive function is associated with years spent in education; individuals who have spent fewer years in education are more likely to be affected. Increased years spent in education may act as protection against cognitive dysfunction in individuals exposed to organic solvents $(12,14,17)$.

Commonly used methods for the treatment of chronic benzene poisoning included the following: cessation of benzene exposure, general supportive therapy, antibiotics, vitamins, corticosteroids, androgens, colony stimulating factors (G-CSF, GM-CSF), blood component therapy, and traditional Chinese medicine. The complications following chronic benzene poisoning includes aplastic anemia, fetal congenital malformation, etc.

Few reports have used magnetic resonance imaging (MRI) to explore the effect of damaged brain regions on cognitive function in patients with chronic benzene poisoning. In this study, we presented a case report of cognitive impairment caused by long-term exposure to chemical dyes that contained benzene. Following timely symptomatic treatment, this patient's cognitive function significantly improved. The aim of this paper was to highlight the systemic damage caused by chronic benzene poisoning, particularly to the central nervous system. Early detection of benzene poisoning is required for the timely diagnosis and treatment of these patients, which then reduces the suffering of those affected.

We present the following article in accordance with the CARE reporting checklist (available at http://dx.doi. org/10.21037/atm-20-6597).

\section{Case presentation}

All procedures performed in studies involving human participants were in accordance with the ethical standards of the institutional and/or national research committee(s) and with the Helsinki Declaration (as revised in 2013). Written informed consent was obtained from the patient for publication of this manuscript and any accompanying images.

\section{The course of the disease}

The patient was a 41-year-old female painter who presented to the psychiatric clinic with cognitive impairment that manifested in the following ways: an inability to complete daily work in the correct order; she always forgot salt when cooking; and she had trouble finding her way home, which concerned her family. Her husband told us that the patient could no longer do things that she used to do well. In addition, she had symptoms such as loss of appetite, nausea and fatigue.

The patient had developed loss of appetite and fatigue two months prior to presentation and was diagnosed with influenza at the local county hospital due to these symptoms and abnormal routine blood test results; her white blood cell count was reduced and her lymphocyte count was slightly increased. Following symptomatic treatment with common cold medication, her gastrointestinal symptoms and fatigue did not be significantly improved. Despite this, the patient continued to work.

One month prior to presentation, the patient's family realized she had developed memory problems. In her work, the patient painted swivel chairs and was meant to paint both sides of each chair. However, she was only painting one side and forgetting to paint the other. She had been doing this work for more than five years. More seriously, the patient's family also found she could not complete housework in the correct order. For example, she always forgot to add salt when cooking. The change most concerning for the patient's husband was that she could no longer find her way home. As a result, on one occasion, she 
Table 1 Patient cognitive function assessments

\begin{tabular}{lccc}
\hline Characteristic & MMSE & MOCA & ADAS-cog \\
\hline Baseline & 7 & 5 & 31.66 \\
At 2 weeks & 19 & 16 & 25 \\
At 3 months & 28 & 22 & 12.34 \\
\hline
\end{tabular}

Table 2 Summary of ADAS-cog tasks

\begin{tabular}{lccc}
\hline Task & Baseline & At 2 weeks & At 3 months \\
\hline Word recall & 7.33 & 6.33 & 4.67 \\
Naming objects and fingers & 1 & 1 & 0 \\
Commands & 2 & 1 & 1 \\
Constructional praxis & 1 & 1 & 1 \\
Ideational praxis & 1 & 1 & 0 \\
Orientation & 5 & 2 & 1 \\
Word recognition & 8.33 & 7.67 & 2.67 \\
Language & 3 & 2 & 0 \\
Comprehension of spoken & 1 & 1 & 1 \\
language & & 1 & 1 \\
Word finding difficulty & 1 & 1 & 0 \\
Remembering test & 1 & 25 & 12.34 \\
instructions & & & \\
Total points & 31.66 & & \\
\hline
\end{tabular}

Table 3 Detection of autoimmune encephalitis (CSF)

\begin{tabular}{lcc}
\hline Characteristic & Result & Reference range \\
\hline Anti-NMDAR IgG & $(-)$ & $(-)$ \\
Anti-AMPA1R IgG & $(-)$ & $(-)$ \\
Anti-AMPA2R IgG & $(-)$ & $(-)$ \\
Anti-LGI 1R IgG & $(-)$ & $(-)$ \\
Anti-CASPR2R IgG & $(-)$ & $(-)$ \\
Anti-GABA BR IgG & $(-)$ & $(-)$ \\
\hline
\end{tabular}

had become lost in the village.

\section{Diagnosis and treatment process}

The patient was brought by her husband to the local county hospital where she received a thyroid function examination. This indicated the patient had hypothyroidism, and she
Table 4 Detection of AD markers in CSF

\begin{tabular}{lccc}
\hline Characteristic & Result & Age $(\mathrm{y})$ & Reference range $(\mathrm{pg} / \mathrm{mL})$ \\
\hline $\mathrm{A} \beta[1-42]$ & $1,273.4 \uparrow$ & $21-50$ & $792 \pm 182$ \\
& & $51-70$ & $790 \pm 228$ \\
& & $>71$ & $797 \pm 230$ \\
T-Tau & $249.5 \uparrow$ & $21-50$ & $136 \pm 89$ \\
& & $51-70$ & $243 \pm 127$ \\
& & $>71$ & $341 \pm 171$ \\
P-Tau & 38.37 & $18-44$ & $19.66-45.67$ \\
& & $45-77$ & $35.84-66.26$ \\
\hline
\end{tabular}

The patient's CSF examinations, which included testing for autoimmune encephalitis (Table 3) and AD markers (Table 4), showed no significant abnormalities to indicate autoimmune encephalitis. The patient did, however, show elevated levels of the $A D$ biomarkers $A \beta$ [1-42] and t-tau. Previous studies have shown that increased levels of $A \beta$ [1-42] (18) and tau $(19,20)$ are the pathological basis of $A D$ and are required for an $A D$ diagnosis. This result indicates that the patient's decline in cognitive function had a specific pathological basis. CSF, cerebrospinal fluid.

was given endocrine therapy (50 $\mu \mathrm{g}$ Euthyrox per day) to improve her thyroid function. However, the patient's symptoms did not improve after nearly two weeks of therapy. As the patient could not adequately perform her work duties, she had to cease working. As her disease progressed, the patient's doctor suggested she attend a more reputable hospital for further treatment.

The patient received treatment in the endocrinology department of our hospital in 2018.04.14 for approximately two weeks before we met her. She had been diagnosed with leukopenia and administered Leucogen tablets $(20 \mathrm{mg}$ three times a day) to increase her white blood cell count. At this time, the patient's thyroid function was normal, but her cognitive function was notably impaired.

We evaluated her cognitive function with a series of tasks (Tables 1,2), and performed a physical examination, a mental examination, a cranial MRI, and a cerebrospinal fluid (CSF) examination (Tables 3,4). Examinations of her physical health and nervous system indicated the patient's vital signs were stable. But for slight hyperreflexia of the tendons, her results were normal. In the mental examination, the patient could only answer the doctor's questions using one or two simple words. For example, when we asked the patient why she came to hospital, she said "cold". When we asked questions like "how are you feeling now" or "what's 

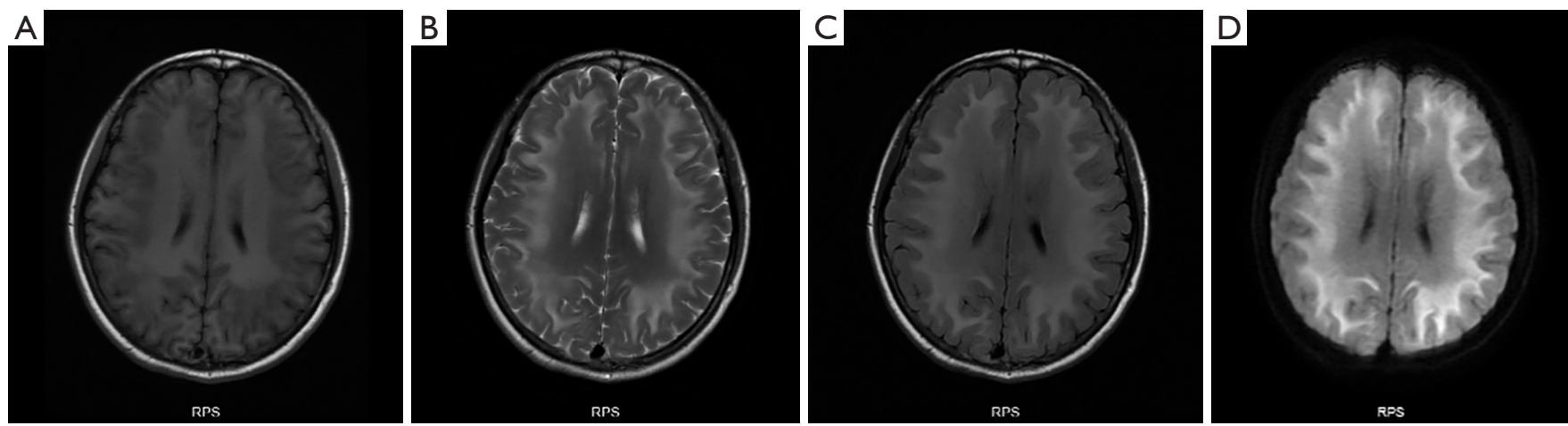

Figure 1 Patient pre-treatment MRI. (A) T1WI showed slightly low signal intensity in the frontal lobe, temporal lobe, occipital lobe, basal ganglia, subcortical white matter, and external capsule. (B) T2WI showed mild brain edema, a slightly shallow sulcus, extensive and patchy high signal intensity, and blurred boundaries in the frontal lobe, temporal lobe, occipital lobe, basal ganglia, and internal capsule. (C) FLAIR showed extensive and patchy high signal intensity in the frontal lobe, temporal lobe, occipital lobe, basal ganglia, and internal capsule. (D) DWI showed extensive and patchy high signal intensity in the frontal lobe, temporal lobe, occipital lobe, basal ganglia, and internal capsule. Abnormal signals across large areas were observed in the white matter of the frontal and parietal lobes in both hemispheres. MRI, magnetic resonance imaging.

wrong with you", she was silent. When we asked her what day it was, the patient said, "I don't know". The patient's mental examination indicated she was passive, emotionally apathetic, exhibited slow thinking speed, and had poor thought content. As she showed no psychotic symptoms, such as hallucinations or delusions, and there were no other obvious symptoms, such as decreased interest or insufficient energy, her impairment had gone unnoticed and progressed rapidly. After consulting with the patient and reviewing her medical history, we excluded depressive disorders, schizophrenia, and other mental disorders. Her cranial MRI, however, indicated white matter signal abnormalities (Figure 1).

After a comprehensive analysis of her mental examination results, MRI, medical history, and disease characteristics, and after excluding other diseases, we believed the patient's cognitive impairment might be due to benzene poisoning. We recommended the patient be treated in the Department of Neurology, where she was diagnosed with cognitive decline caused by chronic toxic encephalopathy. Throughout her hospitalization, the patient received citicoline ( $0.1 \mathrm{~g}$ three times a day) and mecobalamin tablets (500 $\mu \mathrm{g}$ three times a day) for the reversal of nerve injury, as well as nerve nourishment, fursultiamine tablets $(25 \mathrm{mg}$ three times a day) and vitamin B1 (10 mg three times a day) to improve appetite, prednisone tablets $(20 \mathrm{mg}$ once a day) as an anti-inflammatory, and hyperbaric oxygen to improve brain function and healing. The patient was also treated with potassium supplements, strengthened nutrition strategies, and other supportive therapies. She also received cognitive training, which included reading every day and talking with her family.

The patient was hospitalized in the Department of Neurology for two weeks, after which her condition improved and her cognitive function somewhat improved. She could actively talk to others, her mood improved, and she could perform daily life activities. When we saw the patient at this time, she could answer our questions with reasonable accuracy and actively spoke with us. The patient told us she felt better and her mind had begun to work. When we asked how she had felt two months ago, she said, "I felt like my brain was rusty and could not work, and nor could I think. I did not know what was wrong with me." The patient added, "Our workshop is about 20 meters squared, but there is only one window. The oil paint smelled terrible. We workers usually had chest tightness and dizziness. There were two others besides me in the workshop who had the same symptoms, and they also stopped working to seek treatment." At this time, we assessed the patient's cognitive function (Tables 1,2) again and she showed notable improvements.

The patient was required to continue receiving treatment after discharge and also received regular outpatient visits. She took her medications every day and received 

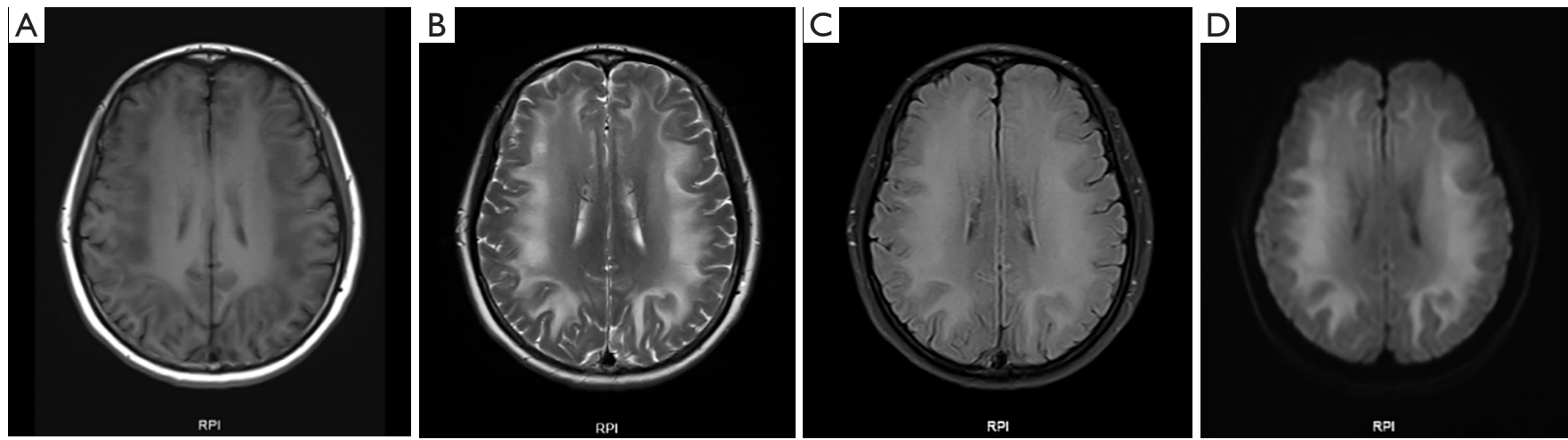

Figure 2 Patient MRI after 1 month of treatment. (A) The low signal intensity previously observed on T1W1 was slightly alleviated. (B) The brain edema previously observed on T2WI was slightly alleviated, and the sulcus was slightly deeper. (C) The high signal intensity observed on FLAIR was slightly decreased. The possibility of white matter demyelination was considered. (D) The high signal shadow previously observed on DWI was slightly alleviated, and the range of abnormal signals was reduced. MRI, magnetic resonance imaging.

hyperbaric oxygen treatment once a week. We performed another cranial MRI (Figure 2) after a month of treatment. Compared with her pre-treatment MRI, these results indicated that the demyelination had slightly improved in the corresponding brain regions.

\section{Outcome of the disease}

Three months following discharge, the patient showed significant improvement. She could look after her children, do housework, and had no notable abnormal contact or communication with others. The patient's emotional state was stable. We assessed her cognitive function (Tables 1,2) again at this time and found it had almost returned to normal.

\section{Discussion}

Chronic benzene poisoning is a serious disease that can severely damage multiple organs. Many studies have explored the effects of chronic benzene poisoning on the hematopoietic system. Hematological diseases, such as cytopenia, aplastic anemia, leukopenia, and leukemia, are frequently associated with benzene poisoning $(7,8)$.

Low levels of benzene exposure $(<1 \mathrm{ppm})$ can affect the hematopoietic system. However, the threshold for causing damage is unknown. An epidemiological study claimed that peripheral blood white blood cell counts and platelet counts were significantly lower in workers exposed to air levels of benzene as low as $1 \mathrm{ppm}$ compared with workers in a control group (21). Benzene exposure may affect the formation and differentiation of various hematopoietic progenitor cells and erythroid colony-forming units in bone marrow. These effects are primarily characterized by decreased peripheral blood cell counts in the early stages of benzene exposure (7).

In this case report, the patient's peripheral blood leukocyte count was slightly decreased. Although she received symptomatic treatment, which included drugs to increase her leukocyte levels, her symptoms of poisoning did not improve. Due to her prolonged exposure to paint that contained benzene, the patient suffered damage to her central nervous system and cognitive function.

Our cognitive function assessments showed that the patient had severe cognitive impairment [mini-mental state examination $(\mathrm{MMSE})=7 / 30$; montreal cognitive assessment $(\mathrm{MOCA})=5 / 30$; Alzheimer's disease assessment scale-cognitive subscale (ADAS-cog) $=31.66$ ] before receiving symptomatic treatment. The patient had serious problems with orientation, short-term memory, attention, language, and visuospatial functions. She could not do housework or perform her work normally and her daily life was significantly affected. After two weeks of symptomatic treatment in hospital, the patient's memory, attention, language, and visuospatial function had somewhat improved. She could control her own diet and communicate with others in a relatively smooth manner. Her state of mind was also improving. After approximately three months of treatment, the patient's cognitive function assessments indicated significant improvement (MMSE increased by $300 \%$; MOCA increased by $340 \%$; ADAS-cog decreased by 61\%). The patient's ADAS-cog results are shown in Table 2. The patient's word recall, word recognition, and language 


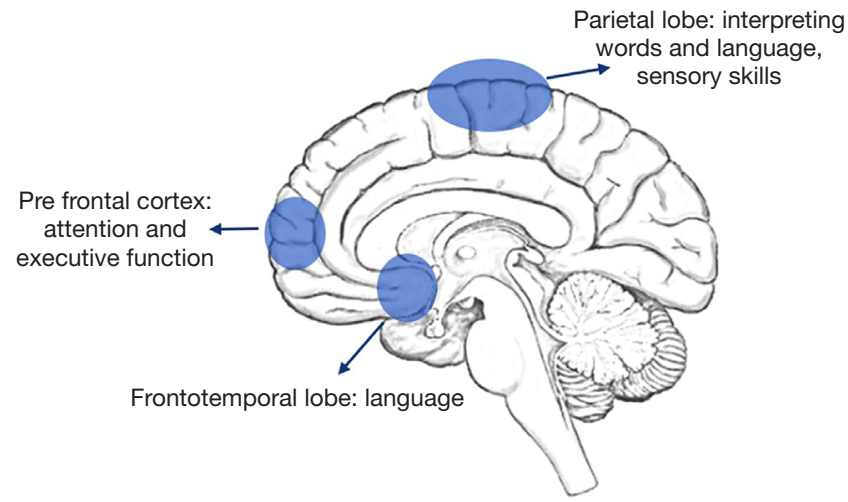

Figure 3 Functions of brain regions related to benzene poisoning pathogenesis.

expression showed particularly significant improvement. This indicates her treatment was effective.

As the patient had cognitive impairment, recent memory loss, and autonomic nerve dysfunction, as well as other symptoms, we tested her CSF for autoimmune encephalitis (Table 3) and CSF markers of Alzheimer's disease (AD) (Table 4). The results showed no significant abnormalities to indicate autoimmune encephalitis, and this disease was excluded. However, as the possibility of earlyonset $\mathrm{AD}$ also had to be excluded, we performed tests for $\mathrm{AD}$-related factors in the patient's CSF and found that her amyloid beta $(\mathrm{A} \beta)$ [1-42] and tau levels were slightly high. However, the brain changes observed in the patient indicated neuronal edema and demyelination caused by long-term benzene exposure. The patient did not show the characteristic atrophy observed in $\mathrm{AD}$, and this disease was therefore excluded. A later review of the patient's clinical manifestations verified this exclusion: her improvements were inconsistent with the irreversibility of $\mathrm{AD}$.

The patient's MRI showed extensive white matter signal abnormalities, particularly in the frontal lobe, temporal lobe, basal ganglia, before treatment. This indicated more extensive injuries to these regions (Figure 3). Studies have shown that the frontal lobe, the anterior region of the cerebral hemispheres, controls movement, language, intelligence, emotional regulation, et cetera (22). The temporal lobe, located beneath the lateral fissure of each cerebral hemisphere is involved with emotion, auditory processing, remembering visuospatial information, et cetera $(22,23)$. The parietal lobe, located at the posterior top of the cerebral hemispheres, controls sensory skills and is important in reading, et cetera (24). The functions of these

\section{Hu et al. Effect of chronic benzene poisoning on cognitive function}

brain regions are consistent with the cognitive dysfunction that the patient exhibited. Compared with her pretreatment MRI, an MRI taken after one month of treatment showed that the white matter signal abnormalities in these regions had reduced. The size of the areas that showed abnormalities had also decreased. These changes aligned with assessments of the patient's symptoms and cognitive function, demonstrating that her treatment was effective.

Due to early detection and treatment of her disease, the patient's social functioning essentially recovered. However, many people are exposed to benzene and other harmful chemicals for extended periods of time due to their work and living environments. Many chronic benzene poisoning patients only receive partial treatment for a range of complex reasons, such as economic factors and health insurance. Some may receive no treatment at all. Additionally, as it has a poor prognosis, this condition is a great burden on affected families and society as a whole. Effective monitoring of harmful gas concentrations in work environments where benzene poisoning can occur fall into this category, providing regular physical examinations and screening of high-risk groups, and reducing the length of time people are exposed to harmful chemicals wherever possible are vitally important measures that must be implemented, in addition to the early detection, diagnosis, and treatment of Chemical solventrelated diseases. The mechanisms by which benzene damages the central nervous system and effective treatment measures must also be explored.

In this case report, the patient had worked in a benzene environment for an extended period. She did not take effective protective measures and she did not have regular health checks. As a result, her body was affected by the benzene exposure, causing clinical symptoms and systemic damage. This case indicates that businesses and governments need to focus more on workers' health to prevent toxic gas exposure. They must improve health education to better equip workers to protect themselves, and they should promote preventative measures rather than treatment measures. In particular, workers should be required to wear gas masks when operating in environments that contain high or even moderate concentrations of benzene. Employers must instruct employees how to prevent benzene absorption through the skin and ensure that eyewash and shower equipment are readily available. In a practical work setting, employers must improve health protection measures for their workers in benzene-polluted environments. They must sensibly arrange production processes, separate benzene operations from benzene-free 
operations, install readily available ventilation detoxification equipment, and regularly monitor air concentrations of benzene to prevent chronic occupational benzene poisoning. Additionally, employers must require employees to receive physical examinations before starting work. Workers whose results indicate they should not participate in operations that involve exposure to toxic or harmful substances should not be permitted perform these duties.

This case report described a patient who suffered from chronic benzene poisoning. There are some controversies about the diagnosis of chronic benzene poisoning, such as how to determine the number of years of occupational exposure, the indicators of changes in the blood system (especially white blood cells), the lack of specificity of bone marrow changes, and so on. However, the general diagnostic criteria for chronic benzene poisoning are as follows: (I) a conclusive history of occupational benzene contact for at least one year; (II) notable changes in hemogram results compared with that obtained preexposure, such as leukopenia; (III) other diseases that could cause the same clinical symptoms are excluded.

There is no "antidote" for benzene poisoning; treatment consists primarily of symptomatic therapies. Similar to leukopenia patients, our case report patient should take drugs to increase her leukocyte levels on a permanent basis. Prevention of benzene poisoning, therefore, should be prioritized above all else. In my opinion, for the treatment of chronic benzene poisoning, causative treatment is necessary, we need to first ensure that the patient is out of the benzene-containing environment.; secondly, symptomatic treatment, we need to actively deal with the clinical symptoms that the patients have already developed.; finally, we need long-term maintenance treatment and follow-up of patients' physical health, provide timely medical intervention.

\section{Acknowledgments}

I would like to thank my tutor for his guidance in the preparation of this paper, as well as the teachers and students of the department for their assistance in writing this paper.

Funding: National Natural Science Foundation of China (No. 81771158).

\section{Footnote}

Reporting Checklist: The authors have completed the CARE reporting checklist. Available at http://dx.doi.org/10.21037/ atm-20-6597

Conflicts of Interest: All authors have completed the ICMJE uniform disclosure form (available at http://dx.doi. org/10.21037/atm-20-6597). The authors report grants from National Natural Science Foundation of China (No. 81771158), during the conduct of the study. The authors have no other conflicts of interest to declare.

Ethical Statement: The authors are accountable for all aspects of the work in ensuring that questions related to the accuracy or integrity of any part of the work are appropriately investigated and resolved. All procedures performed in studies involving human participants were in accordance with the ethical standards of the institutional and/or national research committee(s) and with the Helsinki Declaration (as revised in 2013). Written informed consent was obtained from the patient for publication of this manuscript and any accompanying images.

Open Access Statement: This is an Open Access article distributed in accordance with the Creative Commons Attribution-NonCommercial-NoDerivs 4.0 International License (CC BY-NC-ND 4.0), which permits the noncommercial replication and distribution of the article with the strict proviso that no changes or edits are made and the original work is properly cited (including links to both the formal publication through the relevant DOI and the license). See: https://creativecommons.org/licenses/by-nc-nd/4.0/.

\section{References}

1. Rodriguez B, Yang Y, Guliaev AB, et al. Benzene-derived N2-(4-hydroxyphenyl)-deoxyguanosine adduct: UvrABC incision and its conformation in DNA. Toxicol Lett 2010;193:26-32.

2. Salahinejad M, Ghasemi JB. 3D-QSAR studies on the toxicity of substituted benzenes to Tetrahymena pyriformis: CoMFA, CoMSIA and VolSurf approaches. Ecotoxicol Environ Saf 2014;105:128-34.

3. Capleton AC, Levy LS. An overview of occupational benzene exposures and occupational exposure limits in Europe and North America. Chem Biol Interact 2005;153154:43-53.

4. Hosgood HD, 3rd, Zhang L, Shen M, et al. Association between genetic variants in VEGF, ERCC3 and occupational benzene haematotoxicity. Occup Environ 


\section{Page 8 of 8}

Med 2009;66:848-53.

5. Sorahan T. Occupational benzene exposure and lymphoma risks. Environ Health Perspect 2011;119:A468; author reply A-9.

6. White RF, Proctor SP. Solvents and neurotoxicity. Lancet 1997;349:1239-43.

7. Ahmad Khan H. Short Review: Benzene's toxicity: a consolidated short review of human and animal studies. Hum Exp Toxicol 2007;26:677-85.

8. Smith MT. Advances in Understanding Benzene Health Effects and Susceptibility. Annu Rev Public Health 2010;31:133-48.

9. Daniell WE, Claypoole KH, Checkoway H, et al. Neuropsychological function in retired workers with previous long-term occupational exposure to solvents. Occup Environ Med 1999;56:93-105.

10. Ye C, Lin L, Zhang P, et al. The protective effect of PK11195 on D-galactose-induced amnestic mild cognitive impairment in rats. Ann Transl Med 2020;8:1190.

11. Park RM, Schulte PA, Bowman JD, et al. Potential occupational risks for neurodegenerative diseases. Am J Ind Med 2005;48:63-77.

12. Dick FD. Solvent neurotoxicity. Occupational and Environmental Medicine 2006;63:221-6.

13. Baker EL. Organic solvent neurotoxicity. Annu Rev Public Health 1988;9:223-32.

14. Meyer-Baron M, Blaszkewicz M, Henke H, et al. The impact of solvent mixtures on neurobehavioral performance-Conclusions from epidemiological data. Neuro Toxicology 2008;29:349-60.

15. Gregersen P. Neurotoxic Effects of Organic Solvents in Exposed Workers: Two Controlled Follow-up Studies

Cite this article as: $\mathrm{Hu} \mathrm{J}, \mathrm{Yu} \mathrm{E}$, Liao Z. Changes in cognitive function and related brain regions in chronic benzene poisoning: a case report. Ann Transl Med 2021;9(1):81. doi: 10.21037/atm-20-6597
Hu et al. Effect of chronic benzene poisoning on cognitive function

After 5.5 and 10.6 Years. Am J Ind Med 1988;14:681-701.

16. Edling C, Ekberg K, Ahlborg G Jr, et al. Long-term follow up of workers exposed to solvents. Br J Ind Med 1990;47:75-82.

17. Berr C, Vercambre MN, Bonenfant S, et al. Occupational Exposure to Solvents and Cognitive Performance in the GAZEL Cohort: Preliminary Results. Dement Geriatr Cogn Disord 2010;30:12-9.

18. DeVos SL, Corjuc BT, Commins C, et al. Tau reduction in the presence of amyloid-beta prevents tau pathology and neuronal death in vivo. Brain 2018;141:2194-212.

19. Lacosta AM, Insua D, Badi H, et al. Neurofibrillary Tangles of Abetax-40 in Alzheimer's Disease Brains. J Alzheimers Dis 2017;58:661-7.

20. Hyman BT, Phelps CH, Beach TG, et al. National Institute on Aging-Alzheimer's Association guidelines for the neuropathologic assessment of Alzheimer's disease. Alzheimers Dement 2012;8:1-13.

21. Lan Q, Zhang L, Li G, et al. Hematotoxicity in workers exposed to low levels of benzene. Science 2004;306:1774-6.

22. Vaidya AR, Fellows LK. Ventromedial frontal lobe damage affects interpretation, not exploration, of emotional facial expressions. Cortex 2019;113:312-28.

23. Mirman D, Graziano KM. Damage to temporo-parietal cortex decreases incidental activation of thematic relations during spoken word comprehension. Neuropsychologia 2012;50:1990-7.

24. Cavaco S, Anderson SW, Chen KH, et al. Parietal damage impairs learning of a visuomotor tracking skill. Neuropsychologia 2015;79:106-12.

(English Language Editor: B. Madden) 